\title{
Highly Miniaturized Wideband Negative Group Delay Circuit Using Effective Negative Dielectric permittivity Stopband Microstrip Lines
}

\author{
H. Taher \\ Electronic engineering department \\ National university of Ireland, \\ Maynooth, Ireland \\ htaher@eeng.nuim.ie
}

\author{
R. Farrell \\ Electronic engineering department \\ National university of Ireland, \\ Maynooth, Ireland \\ rfarrell@eeng.nuim.ie
}

\begin{abstract}
In this paper, a effective negative dielectric permittivity $(\epsilon)$ phenomenon is used to obtain compact negative group delay circuit (NGDC). Rectangular complementary split ring resonator (RCSRR) etched on the ground of microstrip line (MSL) is utilized to stimulate this behaviour. Firstly, the desired location of the target delay $\left(f_{0}\right)$ is solely determined using the unit dimensions. Then, the target GD is fully controlled using external resistor $(R)$ connected across the external rectangle of the previously determined RCSRR. Design curves of the proposed structure are established and employed to design NGDC with GD of $-5 \mathrm{~ns}$ at $f_{0}$ of $3.5 \mathrm{GHz}$. The designed NGDC accomplishes $62 \%$ size diminution compared to the smallest circuit size that has ever been achieved before. Moreover, $0.30 \mathrm{GHz}$ zero NGD bandwidth (BW) is achieved. Excellent agreement is noticed between the simulation results of the proposed design and the measurements of the fabricated circuit.
\end{abstract}

Keywords - Microstrip transmission line, negative- $\epsilon$ material, complementary split ring resonators, negative group delay circuit (NGDC).

\section{INTRODUCTION}

The fast evolving of radio frequency (RF) and microwave technology requires different passive and active circuits/components with stringent requirements. Circuit area compactness is one of the most rigorous design specifications for the nowadays systems.

Negative group velocity, and consequently NGD, is an example of abnormal wave propagation phenomena and it could be observed within the limited frequency band of signal in certain media under signal attenuation condition [1]. This phenomenon is exploited to build NGDCs which are needed in diverse applications and systems such as efficiency enhancement of feedforward linearization [2], beam-squit minimization in phased array antenna systems [3], and eliminating phase variation with frequency in phase shifters [4].

NGDC implementation basically could be classified into two categories, namely, passive [5] and active [6]. Using active NGDC adds a noise to the system and consequently the overall signal to noise ratio of the system is deteriorated. Regarding the later class, passive NGDC are realized using many techniques, such as, external RLC resonators [7], without external RLC resonators [8]-[9] periodic structures [10] and MSL [11]. All these designs use either quarter wave length lines or lumped elements as resonators. Consequently, they suffer from large area circuits as well as the small bandwidth. No concentration is paid to solve the aforementioned issues. The most efforts are focused to solve high signal attenuation (SA) or multiband operation problems. Nevertheless, only few researches use defected planer structures, U- Shape defected ground structure (DGS), to achieve compact NGDC area [12]-[13]. However, the achieved figures of area size reduction and the accompanied BW still need to drastic improvement. Furthermore, the adopted solution to increase the operating bandwidth is to cascade couple of units together. As a result, large size area circuits are fabricated.

On the other hand, there is a growing interest in using CSRR as constituent particles for the design of novel planar microwave components [14]. The main advantage of utilizing CSRR is that they are significantly smaller in size than conventional resonator structures enabling the design of very compact circuits. CSRR is excited by means of an axial time varying electric field, which is concentrated between the conductor line and ground plane. As a result, a negative $\epsilon$ is obtained in specified frequency band and the structure exhibits signal attenuation condition which is needed to acquire the NGD phenomenon functionality.

Based on the mentioned above, this work employs RCSRR to build extremely miniaturized microstrip NGDC. To this end, detailed design curves are established to get the physical dimensions of the RCSRR and the value of $R$ which are needed to achieve the target NGD value at the required $f_{0}$.

This paper is organized as follows, description of the demonstrated NGDC is elaborated in section two. Design, implementation and verification of the developed NGDC are presented in section three. Finally, the conclusion is drawn in section four.

\section{RCSRR BASED NGDC}

. Fig. 1 shows the structure of the proposed NGDC which comprises MSL and RCSRR etched in the ground plane. The MSL has width (W) and length (L) respectively. Moreover, there are input and output $50 \Omega$-lines ports have width $\left(\mathrm{W}_{0}\right)$ and length $\left(\mathrm{L}_{0}\right)$. The outer dimensions of the RCSRR are as long as 
the corresponding values of the hosting MSL, W and L. Furthermore, to keep the design procedure simple as much as possible, the etched gap widths $g, c$ and $d$ values are set equal. On the other hand, $R$ is harnessed as tuning component of the required NGD value.

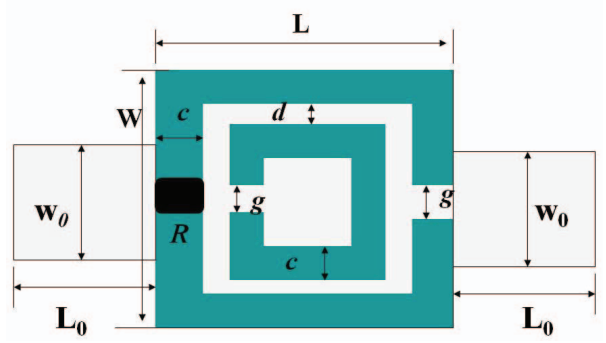

Fig. 1. The proposed NGDC structure

The utilized substrate in all simulations is RT/Duroid 5880 with a dielectric constant of 2.2 and thickness of $0.79 \mathrm{~mm}$. The values of $\mathrm{W}_{0}$ and $\mathrm{L}_{0}$ are fixed to $2.4 \mathrm{~mm}$ and $3 \mathrm{~mm}$ respectively. To verify the occurrence of NGD phenomenon using the presented structure, NGDC with the following dimensions: $\mathrm{L}=14 \mathrm{~mm}, \mathrm{~W}=5.4 \mathrm{~mm}$ and $g=0.3 \mathrm{~mm}$ is simulated. Regarding $R$, it is not used in the simulation to show the capability of RCSRR to produce NGDC. All the simulations are carried out using time-domain finite integration technique using CST microwave studio package. There are many transmission zeroes occurred concurrently and this is attributed to quasi static resonance for the first dip $\left(f_{0}\right)$ and dynamic resonance for the others [15]. Only the NGD engendered by the first zero is considered.

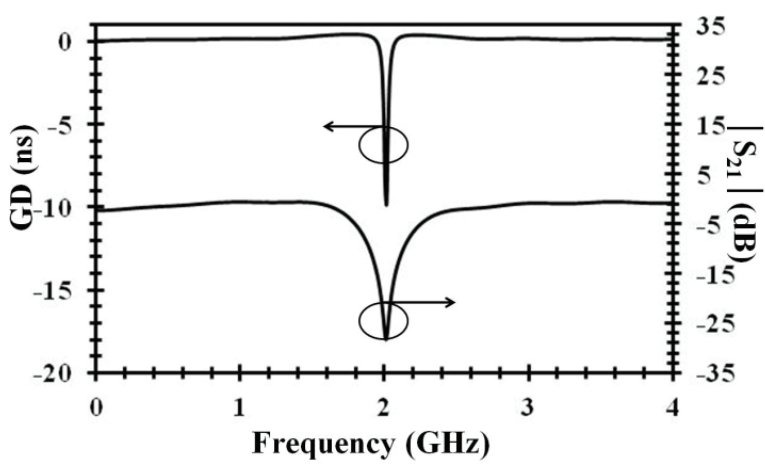

Fig. 2. Simulation results of RCSRR unit, without using external R.

Existence of NGD phenomenon around the resonance of RCSRR is obviously shown in Fig.2. The obtained NGD value is $-9.5 \mathrm{~ns}$ at $f_{0}$ of $2.0 \mathrm{GHz}$. The dependence of the obtained NGD on $R$ as well as their locations on geometrical dimensions of RCSRR are investigated, ploted as design curves and presented in the next section.

\section{NGDC DESIGN AND IMPLEMENTATION}

Design curves technique is the adopted methodology to design the target NGDC. To establish them, the geometrical dimensions and $R$ are varied, while, $f_{0}$ and GD are listed and plotted. With regard to geometrical dimensions, $g$ is varied from $0.2 \mathrm{~mm}$ to $0.6 \mathrm{~mm}, \mathrm{~W}$ is chosen to vary between $2.8 \mathrm{~mm}$ and $6 \mathrm{~mm}$, and finally, $\mathrm{L}$ is altered from $6 \mathrm{~mm}$ to $16 \mathrm{~mm}$. On the other hand, $R$ is varied from $3 \mathrm{k} \Omega$ to $15 \mathrm{k} \Omega$. Two different sets of curves are aimed. Concerning the first set, $f_{0}$ values are plotted versus various values of the geometrical dimensions of the NGDC structure. Regarding the second one, GD values are plotted versus different values of $R$ at specific values of geometrical dimensions.

Three plots among the obtained design curves for $f_{0}$ are shown in Figs. 3-5. The observed values of $f_{0}$ are plotted versus $\mathrm{L}$ for different values of $\mathrm{W}$, at $g$ equals $0.6 \mathrm{~mm}, 0.4 \mathrm{~mm}$ and $0.2 \mathrm{~mm}$ respectively. It is noted that, at particular combination of $\mathrm{W}$ and $\mathrm{L}$ the resulting $f_{0}$ values are decreased as $g$ values get down. The reason is as $g$ is decreased, the equivalent capacitance of the structure is increased and consequently $f_{0}$ value is lowered. Consequently, circuit miniaturization could be possible by decreasing value of $g$. Besides, as $g$ is decreased, smaller values of $\mathrm{W}$ are attainable which it allows for more solutions at specific $f_{0}$ to be realized. On other side, $g$ equals to $0.2 \mathrm{~mm}$ is selected as the smallest value because the available fabrication tools cannot realize gap widths less than this amount.

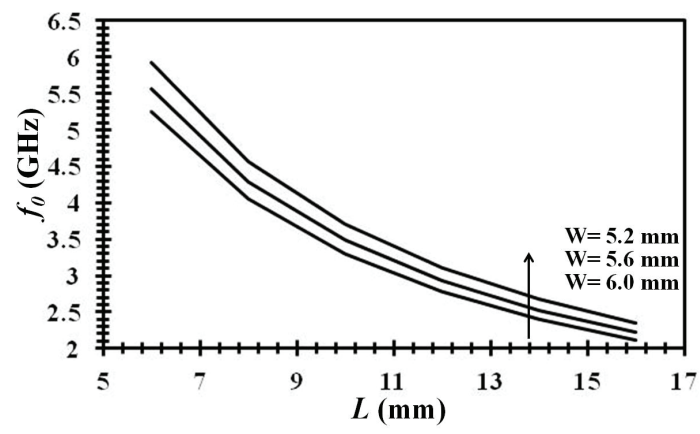

Fig. 3. Variation of $f_{0}$ versus $\mathrm{L}$ for different values of $\mathrm{W}$ at $g=0.6 \mathrm{~mm}$.

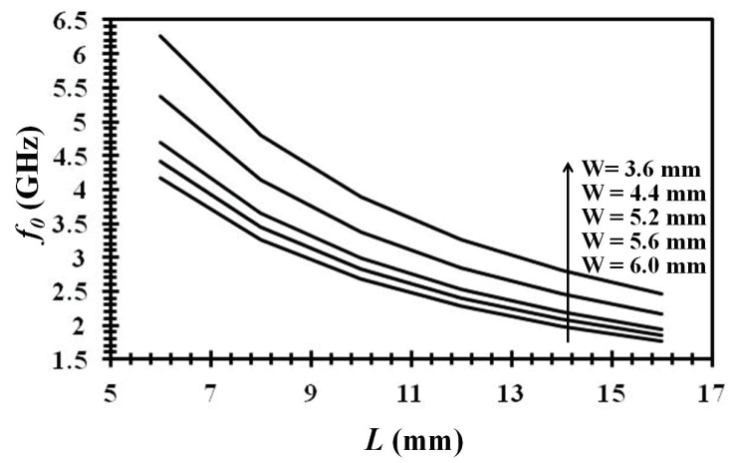

Fig. 4. Variation of $f_{0}$ versus $\mathrm{L}$ for different values of $\mathrm{W}$ at $g=0.4 \mathrm{~mm}$.

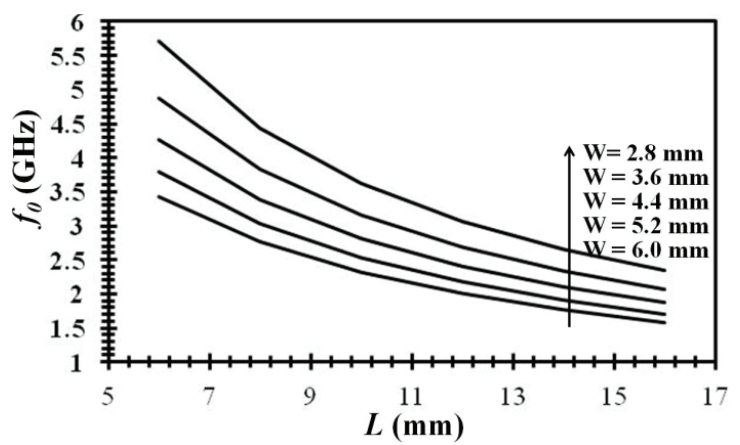

Fig. 5. Variation of $f_{0}$ versus $\mathrm{L}$ for different values of $\mathrm{W}$ at $g=0.2 \mathrm{~mm}$. 
Concerning the design curves of the GD, a lot of design curves are generated, couple of them are depicted in Figs. 6-7. Each design curve plots the obtained NGD for many values of $\mathrm{L}$ at specific $\mathrm{W}$ and $g$. For example, NGD values range from $1.52 \mathrm{~ns}$ to $-13.15 \mathrm{~ns}$ could be achieved using various values of $\mathrm{L}$ and $R$, as depicted in Fig. 6 . It is noticed that, as $R$ and $\mathrm{L}$ are augmented, the achieved NGD value is raised. Any change in $g$ value does not affect the aforementioned overall performance. The only difference is that the obtained GD values range is slightly diminished. For instance, if $g$ value is lowered to 0.2 $\mathrm{mm}$ and $\mathrm{W}$ value is maintained at $6 \mathrm{~mm}$, the acquired values of $\mathrm{GD}$, in that case, take values in the range of $-1.75 \mathrm{~ns}$ to -11.51 ns, as shown in Fig.7.

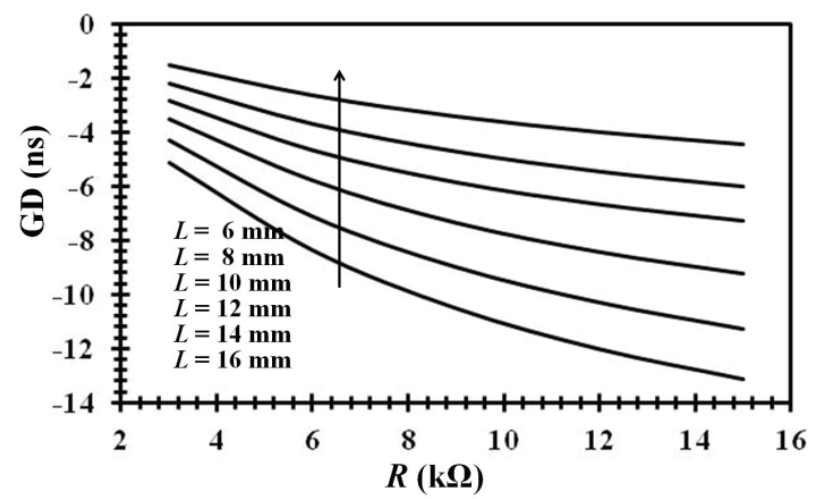

Fig. 6. Variation of GD versus $R$, for different values of $\mathrm{L}$, at $g=0.6 \mathrm{~mm}$ and $\mathrm{W}=6 \mathrm{~mm}$

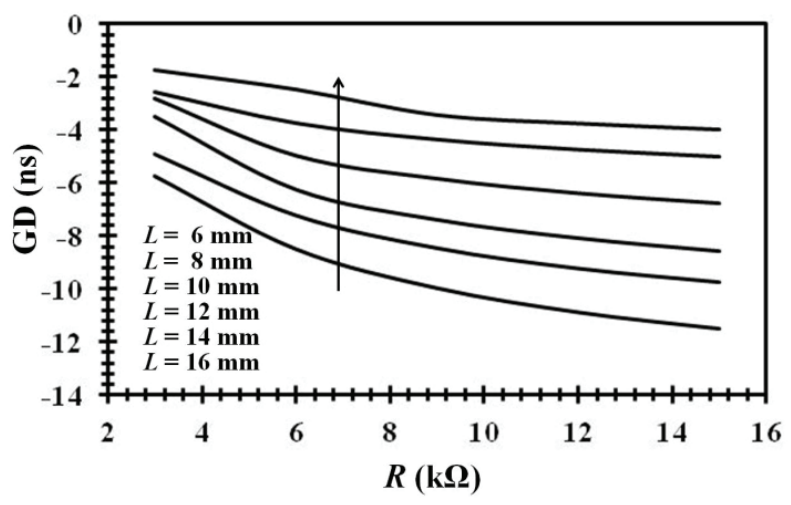

Fig. 7. Variation of GD versus $R$, for different values of $\mathrm{L}$, at $g=0.2 \mathrm{~mm}$ and $\mathrm{W}=6 \mathrm{~mm}$.

The goal is to design the GD of -5 ns for the worldwide interoperability for microwave access (WiMax) band operating at $f_{0}$ of $3.50 \mathrm{GHz}$.

Firstly, dimensions of the circuit are chosen to obtain the required $f_{0}$ using the first group of the design curves. The aim is to get the target specifications using NGDC having minimum possible area. As shown in Figs 3-5, the target $f_{0}$ could be achieved through different designs, i.e, many combinations of $\mathrm{W}, \mathrm{L}$ and $g$. The layout area is calculated by multiplying $\mathrm{W}$ by L. As it has already been discussed, minimum attainable area at specific $f_{0}$ is accomplished using minimum $g$. Consequently, design curves, shown in Fig. 5, which has $g$ equals to $0.2 \mathrm{~mm}$ is utilized in the design procedure. The minimum obtainable area approximately equals $32.8 \mathrm{~mm}^{2}$ and could be realized using different values of $\mathrm{W}$ and $\mathrm{L}$. Among them, $\mathrm{W}=4.20$ $\mathrm{mm}$ and $\mathrm{L}=7.83 \mathrm{~mm}$ are selected. Then, one GD curve, parameterized in terms of the predetermined geometrical dimensions from the first step, is picked from the design curves of the second set, as shown in Fig. 8. Utilizing the selected curve, it is found that $R$ equals to $11.5 \mathrm{k} \Omega$ realizes the required $-5 \mathrm{~ns}$.

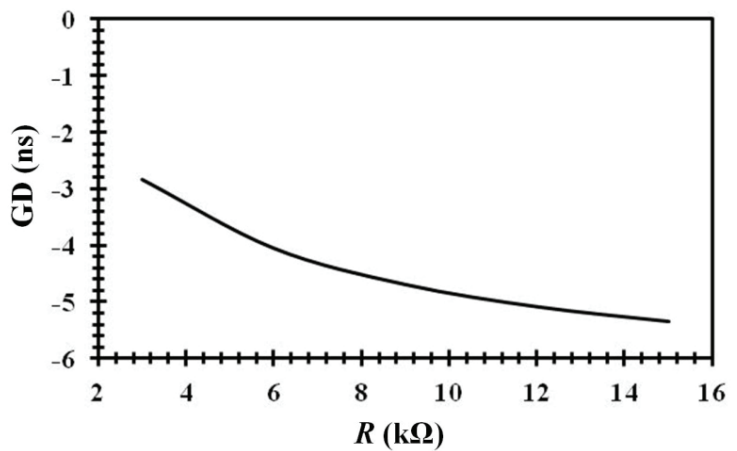

Fig. 8. Variation of GD versus $R$, at $\mathrm{g}=0.2 \mathrm{~mm}, \mathrm{~W}=4.20 \mathrm{~mm}$ and $\mathrm{L}=$ $7.83 \mathrm{~mm}$.

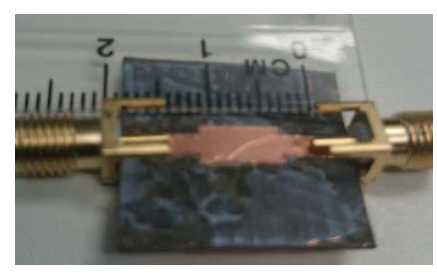

a

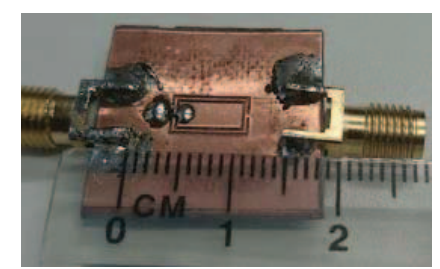

b
Fig. 9. Photograph of the fabricated NGDC, a) Top and b) Bottom.

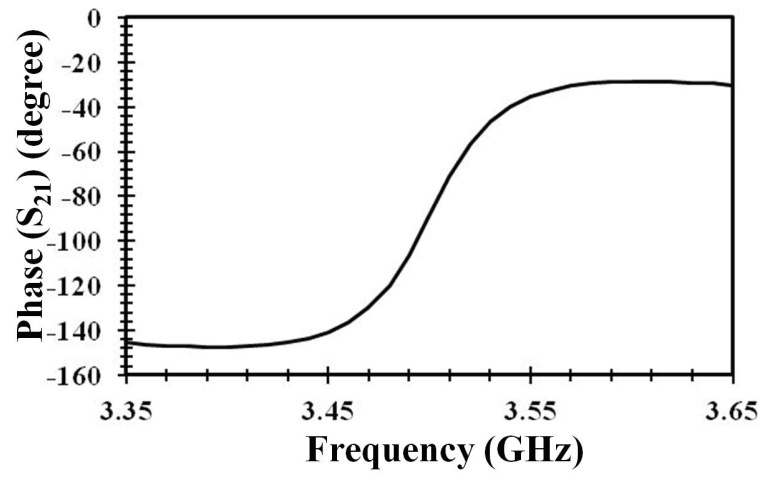

Fig. 10. Measured phase response.

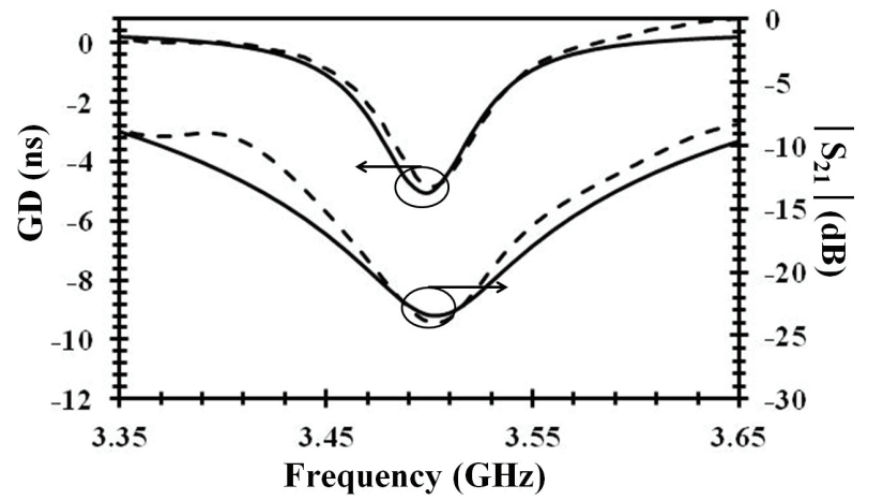

Fig. 11. Comparison between simulated (-) and measured (- - ) GD and signal attenuation, $|\mathrm{S} 21|$. 
To validate the design, NGDC is fabricated using standard printed circuit board technology; the fabricated circuit is shown in Fig.9. The measured phase, as depicted in Fig. 10, exhibits positive slope behaviour which it confirms the negative group delay phenomena occurred at the required operating frequency range. On the other hand, comparison shown in Fig. 11 reveals a good agreement between the simulation of the designed NGDC and the measurements of the fabricated NGDC. It is observed that the measured GD of $-4.85 \mathrm{~ns}$ is attained with SA of $24.15 \mathrm{~dB}$ at $f_{0}$ of $3.503 \mathrm{GHz}$. This considerable amount of SA could be easily compensated using single subsequent amplification stage. Regarding the frequency behaviour, the achieved BW amounts $300 \mathrm{MHz}$. To assess the competence of the proposed NGDC, its figures of merit are compared with the corresponding quantities of previously published researches.

TABLE I

Performance Comparison of the Proposed NGDC WITH OTHER WORKS

\begin{tabular}{c|c|c|c|c|c|c}
\hline & $\begin{array}{c}\boldsymbol{f}_{\boldsymbol{o}} \\
(\mathbf{G H z})\end{array}$ & $\begin{array}{c}\text { GD } \\
(\mathbf{n s})\end{array}$ & $\begin{array}{c}\text { NGD } \\
\mathbf{B W} \\
(\mathbf{M H z})\end{array}$ & $\begin{array}{c}\text { SA } \\
(\mathbf{d B})\end{array}$ & $\begin{array}{c}\text { No.of } \\
\text { stages }\end{array}$ & $\begin{array}{c}\text { Dimensions } \\
\left(\boldsymbol{\lambda}_{\mathbf{0}}^{\mathbf{2}}\right)\end{array}$ \\
\hline$[8]$ & 2.14 & -7.5 & 40 & 17.45 & 2 & $0.28^{*} 0.5$ \\
\hline$[9]$ & 2.14 & -10 & 120 & 60 & 2 & $0.44^{*} 0.50$ \\
\hline$[11]$ & 1.962 & -6.5 & 200 & 35.2 & 2 & $0.40^{*} 0.40$ \\
\hline$[12]$ & 3.5 & -5 & 200 & 22 & 1 & $0.046^{*} 2.6$ \\
\hline $\begin{array}{c}\text { This } \\
\text { work }\end{array}$ & $\mathbf{3 . 5}$ & $\mathbf{- 5}$ & $\mathbf{3 0 0}$ & $\mathbf{2 5}$ & $\mathbf{1}$ & $\mathbf{0 . 0 5 * 0 . 0 9 1}$ \\
\hline
\end{tabular}

Concerning the area size and as listed in TABLE I, the smallest accomplished NGDC area was presented in [12] and it amounts to $22 \times 4 \mathrm{~mm}^{2}$. However, the proposed RCSRR NGDC occupies only $38 \%$ from this area. Though, it is only single stage, not two, as most other works use, the proposed NGDC exhibits the highest BW value among the other works. Therefore, the demonstrated unit is superior in both of area size and frequency behaviour aspects. Regarding SA, the demonstrated NGDC offers a quite competitive value which is $25 \mathrm{~dB}$. Consequently and unequivocally, the demonstrated NGDC has the best overall performance among the others.

\section{CONCLUSION}

In this paper, extremely miniaturized NGDC using the RCSRR is examined. Due to the attenuation characteristic of the created negative- $\epsilon$ material a NGD phenomenon is occurred at the assigned frequency. On the other hand, utilizing external resistor allow us to control NGD value. Design curves are built and exploited to design the developed NGDC. Furthermore, the designed NGDC is fabricated and verified experimentally. Besides the compact size, the proposed NGDC has wide BW, compared to the previously published results. Moreover, it is easy to design and expected to help to minimize the area size of contemporary wireless communication systems, as example, in beam-squint eliminating circuit of serially fed antennas arrays.

\section{ACKNOWLEDGMENT}

This research is supported by Science Foundation Ireland under Grant No. 10/CE/I1853. The authors appreciatively acknowledge this support. Dr. Hany is also Associate Prof. Researcher at Electronic Research Institute (ERI) -Giza Egypt.

\section{REFERENCES}

[1] M. Mojahedi, K. J. Malloy, G. V. Eleftheriades, J. Woodley, and R. Y. Chiao, "Abnormal wave propagation in passive media," IEEE Journal of Selected Topics in Quantum Electronics, Vol. 9, no. 1, pp. 30-39, April 2003.

[2] H. Choi, Y. Jeong, C. D. Kim, and J. S. Kenney, "Efficiency enhancement of feedforward amplifiers by employing a negative group delay circuit," IEEE Trans. Microw. Theory Tech., vol. 58, no. 5, pp. 1116-1125, May 2010.

[3] S. S. Oh and L. Shafai, "Compensated circuit with characteristics of lossless double negative materials and its application to array antennas," IET Microw. Antennas \& Propagation, vol. 1, no. 1, pp. 29-38, Feb. 2007.

[4] B. Ravelo, M. L. Roy, and A. Perennec, "Application of negative group delay active circuits to the design of broadband and constant phase shifters," Microw. Optical Tech. Letters, vol. 50, no. 12, pp. 3078-3080, Dec. 2008.

[5] H. Choi, Y. Kim, Y. Jeong and C. D. Kim, "Synthesis of reflection type negative group delay circuit using transmission line resonator," Proc. of 39th European Microw. Conference, 902-905, Sept. 2009.

[6] B. Ravelo, A. Perennec, M. L. Roy, and Y. G. Boucher, "Active microwave circuit with negative group delay," IEEE Microw. Wireless Compon. Letters, vol. 17, no. 12, pp. 861-863, Dec. 2007.

[7] H. Choi et al., "A design of composite negative group delay circuit with lower signal attenuation for performance improvement of power amplifiers linearization technique," in IEEE Int. Microw. Symp. Dig., Jun. 2011, pp. 1-4.

[8] G. Chaudhary and Y. Jeong, "Transmission line negative group delay networks with improved signal attenuation," IEEE Antenna and Wireless Propagation Letters, vol. 13, no. 1, pp. 1039-1042, Jul. 2014.

[9] G. Chaudhary and Y. Jeong and J. Lim, "A design of reconfigurable negative group delay circuit without External Resonators," IEEE Antenna and Wireless Propagation Letters, vol. 14,, pp. 883-886, Dec. 2015.

[10] O. F. Sidhiqui, M. Mojahedi, and G. V. Eleftheriades, "Periodically loaded transmission line with effective negative group delay index and negative group velocity," IEEE Trans. Antenna Propag., vol. 51, no. 10, pp. 2619-2625, Oct. 2010.

[11] G. Chaudhary, J. Jeong, P. Kim, Y. Jeong, and Jongsik Lim, "Microstrip line negative group delay filters for microwave circuits," IEEE Trans. Microw. Theory Tech., vol. 62, no. 2, pp. 234-243, Feb. 2014.

[12] G. Chaudhary, J. Jeong, P. Kim, Y. Jeong, and Jongsik Lim, "Compact Negative Group Delay Circuit Using Defected GroundStructure," Proc. of Asia-Pacific Microwave. Conference, 22-24, Nov. 2013.

[13] G. Chaudhary, Y. Jeong, and J. Lim, "Miniaturized dual-band negative group delay circuit using dual-plane defected structures," IEEE Microw. Wireless Compon. Letters., vol. 24, no. 8, pp. 521-523, Feb. 2014.

[14] F. Falcone, T. Lopetegi, J.D. Baena, R. Marqués, F. Martín, and M. Sorolla, "Effective negative- $\epsilon$ stop band microstrip lines based on complementary split ring resonators," IEEE Microw. Wireless Compon. Letters, vol. 14, no. 6, pp. 521-523, June. 2004.

[15] M. K. Mandal, P. Mondal, S. Sanyal, and A. Chakrabarty, "Low Insertion-Loss, Sharp-Rejection and Compact Microstrip Low-Pass Filters," IEEE Trans. Microw. Theory Tech., vol. 16, no. 11, pp. 11161125, Nov.2006. 